\title{
Atomic density effects on temperature characteristics and thermal transport at grain boundaries through a proper bin size selection
}

\author{
Truong Quoc Vo, ${ }^{1}$ Murat Barisik, ${ }^{2, a)}$ and BoHung Kim ${ }^{1, a)}$ \\ ${ }^{1}$ School of Mechanical Engineering, University of Ulsan, Daehak-ro 93, Namgu, Ulsan 680-749, South Korea \\ ${ }^{2}$ Department of Mechanical Engineering, Izmir Institute of Technology, Urla, Izmir 35430, Turkey
}

(Received 29 January 2016; accepted 3 May 2016; published online 20 May 2016)

\begin{abstract}
This study focuses on the proper characterization of temperature profiles across grain boundaries (GBs) in order to calculate the correct interfacial thermal resistance (ITR) and reveal the influence of GB geometries onto thermal transport. The solid-solid interfaces resulting from the orientation difference between the (001), (011), and (111) copper surfaces were investigated. Temperature discontinuities were observed at the boundary of grains due to the phonon mismatch, phonon backscattering, and atomic forces between dissimilar structures at the GBs. We observed that the temperature decreases gradually in the GB area rather than a sharp drop at the interface. As a result, three distinct temperature gradients developed at the GB which were different than the one observed in the bulk solid. This behavior extends a couple molecular diameters into both sides of the interface where we defined a thickness at GB based on the measured temperature profiles for characterization. Results showed dependence on the selection of the bin size used to average the temperature data from the molecular dynamics system. The bin size on the order of the crystal layer spacing was found to present an accurate temperature profile through the GB. We further calculated the GB thickness of various cases by using potential energy (PE) distributions which showed agreement with direct measurements from the temperature profile and validated the proper binning. The variation of grain crystal orientation developed different molecular densities which were characterized by the average atomic surface density (ASD) definition. Our results revealed that the ASD is the primary factor affecting the structural disorders and heat transfer at the solid-solid interfaces. Using a system in which the planes are highly close-packed can enhance the probability of interactions and the degree of overlap between vibrational density of states (VDOS) of atoms forming at interfaces, leading to a reduced ITR. Thus, an accurate understanding of thermal characteristics at the GB can be formulated by selecting a proper bin size. Published by AIP Publishing. [http://dx.doi.org/10.1063/1.4949763]
\end{abstract}

\section{INTRODUCTION}

The reduction of grain size down to the nanometer levels has resulted in increased research in electronic and mechanical devices. ${ }^{1,2}$ With the dimensions of devices approaching the nanometer scale, atomistic geometries such as grain boundaries (GBs) increasingly dominate physical mechanisms due to mismatch of the orientation, translation, and composition between crystals. ${ }^{3}$ Prediction modeling with in-depth understanding is one of the most important ventures to obtain optimal performances in the current nano-industry. It is clear that the behaviors and reliability of nano-devices depend strongly on the way the systems dissipate or absorb heat based on particular application requirements. However, recent studies have revealed that thermal management becomes more difficult as the device size decreases, leading to problems of heat transfer at solid-solid interfaces. ${ }^{1,2}$ These challenges remain to be addressed. Therefore, an accurate understanding of fundamental processes occurring at thermal boundaries is essential to design nano-structured devices aimed at optimizing operational efficiency.

\footnotetext{
a) Authors to whom correspondence should be addressed. Electronic addresses: muratbarisik@iyte.edu.tr, Tel.: +90-232-750-6784 and bohungk@ulsan.ac.kr, Tel.: +82-52-259-2705.
}

Thermal transport through a GB produces a discontinuity of the temperature due to the interfacial thermal resistance (ITR), which is induced by the differences in composition, structure, and energy carrier at the boundary. This resulting temperature jump ${ }^{4}(\Delta T)$ can be expressed as follows:

$$
\Delta T=R_{\mathrm{th}} J,
$$

where $R_{\mathrm{th}}$ is ITR and $J$ is the given or measured heat flux. Even though the ITR is small or negligible in macroscopic problems, it is significant in multi-scale methods or nanoscale devices and structures. The ITR plays a key role in design optimization as these devices are increasingly defined by their boundaries.

There have been several theoretical models developed to estimate the ITR such as the acoustic mismatch model ${ }^{5}$ (AMM) and the diffuse mismatch model ${ }^{6}$ (DMM). However, these two models usually fail to compute heat conduction in a superlattice. ${ }^{7}$ Therefore, a method is needed whereby the thermal resistance at the solid-solid interface can be calculated more specifically. Molecular dynamics (MD) simulation is a potential tool to surmount the restrictions of theoretical models. MD simulation has been widely applied to investigate the thermal transport at GBs. ${ }^{7-20}$ By using MD simulation, it is possible to directly observe the independent molecular 
motion, which has so far been very difficult to obtain through experiments. In addition, the ITR can be interpreted by phonons, which are thermal energy carriers which play an important role when studying nanoscale heat transfer in a superlattice. The behaviors of phonon scattering at interfaces have been very well studied in the past. ${ }^{7,16-25}$ Generally, the energy is totally transmitted in a single or perfect crystal. Meanwhile, the phonons are strongly scattered at the GB, where a significant amount of total energy is reflected due to the discontinuity in the structure between two crystals.

Recently, the ITR has been computationally measured between two media. ${ }^{26-31}$ These reports suggest that density is one of the key factors affecting the thermal resistance at the interface. For instance, an increase of the atomic density at the interface improves the probability of intermolecular interactions, resulting in an enhanced phonon transmission and, consequently, a reduced ITR. Therefore, the atomic density of crystals must be carefully considered when studying heat flow across solid-solid interfaces. The role of density is additionally revealed in the estimation of the GB energy. This energy is the free energy associated with the presence of GB and cannot be ignored when investigating nanoscale thermal transport. Computer simulations have suggested that the GB energies are strongly dependent on dislocations as well as disorder at the interface, which are highly related to the density of crystals. ${ }^{7,20}$ Therefore, it becomes increasingly important to study the crystal atomic density effects in detail to provide a sophisticated understanding and control of heat transfer at the nanoscale.

The temperature jump is calculated as the difference of the temperature right at the GB between two linear fits to the temperature gradient on both sides of the hot and cold walls. Numerous MD studies showed that the temperature drops abruptly at the interface due to the phonon scattering and ITR. ${ }^{7,13-15}$ Meanwhile, it was additionally found that there are other temperature gradients at solid-solid interfaces. ${ }^{10-12}$ These gradients are much steeper than those in the bulk solid. Hence, it is evident that the existence of temperature slopes denotes thermal variations at GBs. We deduce that the differences of the temperature profiles from those references are due to the ambiguity in the bin thickness divided for averaging temperature data. Using an inaccurate bin size may not achieve the correct ITR since the ITR is calculated based on the temperature jump obtained from the temperature distribution. Moreover, having a whole solid material with a perfect single crystal structure seems to be nearly impossible. Different crystal structures always form in a solid domain. At the macro/micro scale, it is reasonable to utilize the given thermodynamic thermal conductivity for further computations or investigations. However, this value is not applicable at the nanoscale. ${ }^{32-36}$ Recently, Hua and Cao studied the ballistic transport induced as some of the phonons directly fly from one boundary to another without scattering when the characteristic length is comparable to the phonon mean free path. ${ }^{37}$ The ballistic transport causes deviations to the classical Fourier's law of heat conduction, for example, atomic geometry dependence of temperature jump and thermal conductivity. Therefore, it is necessary to obtain the accurate temperature profiles across the GB to calculate the correct ITR. Subsequently, these findings can be utilized to accurately estimate the efficiency of nano-devices. The temperature distribution in a system is distinctly observed when fine bins are used rather than coarse bins. However, it is questionable how fine the bins must be since this has not been characterized to date. Many questions remain unanswered and there are a few discrepancies in the published results. Thus, a proper selection of the bin size is essential.

Given these challenges, we dedicate particular attention to the proper characterization of the temperature profiles across the GB in order to obtain the correct ITR. To address this, we provide a proper bin size selection method which can be applied universally. Under such conditions, the effects of GB geometries onto thermal transport will be well characterized. The solid-solid interfaces arising from the orientation difference between the (001), (011), and (111) surface models of FCC copper were investigated in this study. One end of the system is subjected to $280 \mathrm{~K}$, while the other is maintained at $360 \mathrm{~K}$. The material and temperature difference studied here are typically applied in solar thermoelectric generators $^{38}$ and a semiconductor fabrication process. ${ }^{39}$

Our paper is organized as follows. (1) In Section II, we summarize the theoretical background for the heat flux and GB energy calculations in MD, followed by a description of the simulation domain and a summary of the differences of the temperature profiles between solid-liquid and solid-solid interfaces. (2) In Section III, our approach for the bin size selection is described and evaluated by comparison with other studies. (3) In Section IV, we investigate the effects of the GB geometries on the thermal transport at interfaces. In addition, atomic density and temperature characteristics at the GB are elucidated. Finally, (4) in Section V, our findings and conclusions are reported.

\section{THEORETICAL BACKGROUND}

The thermal properties at the solid-solid interface are computed by using a non-equilibrium molecular dynamics (NEMD) simulation method. The main idea of NEMD is to create a heat flux through the system by applying a thermostat to specific regions. The schematic simulation diagram of the nanoscale heat transfer system utilized in the present study is shown in Fig. 1(a). The simulation box was constructed by two walls of solid copper with different oriented structures but similar sizes, which induces a GB at the middle of the box. The total dimensions of each grain in the vertical (X), lateral $(\mathrm{Y})$, and longitudinal (Z) directions are $3 \mathrm{~nm}, 3 \mathrm{~nm}$, and $6 \mathrm{~nm}$, respectively. The embedded atom method (EAM) was applied to model the interatomic forces between the solids. This is because it accurately describes the total energy of a metal by considering the embedding energy as a function of the atomic electron density. ${ }^{40}$

Initially, two grains were located with an interface spacing $\left(\delta d_{\text {initial }}\right)$ calculated from the theoretical arithmetic mean as $\delta d_{\text {initial }}=\left(d_{\mathrm{G} 1}+d_{\mathrm{G} 2}\right) / 2$, where $d_{\mathrm{G} 1}$ and $d_{\mathrm{G} 2}$ are the spacing between adjacent lattice planes in grain 1 and grain 2, respectively. Periodic boundary conditions were applied in the $\mathrm{X}, \mathrm{Y}$, and $\mathrm{Z}$ directions. Energy minimization followed by 


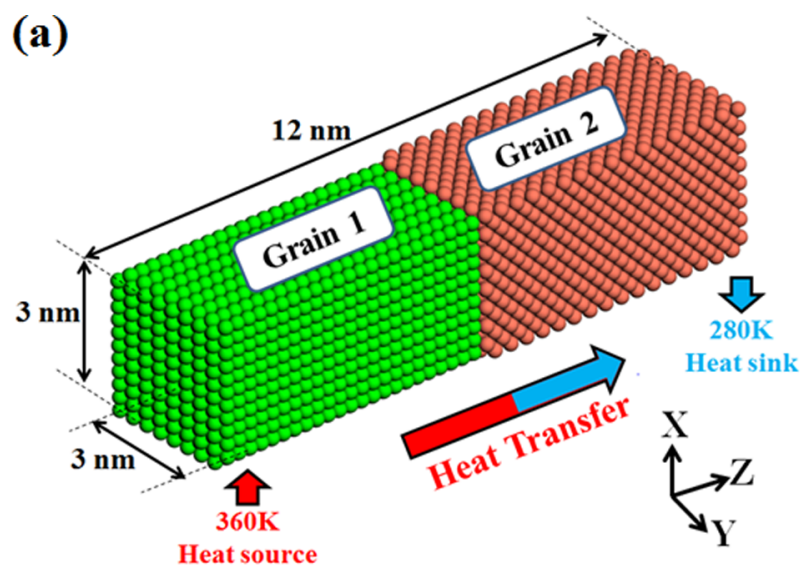

(b)

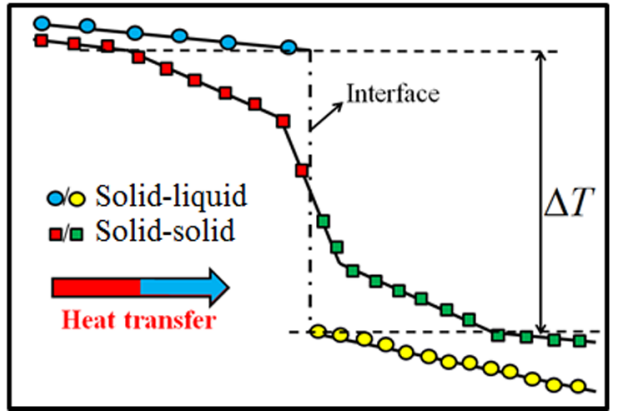

(c)

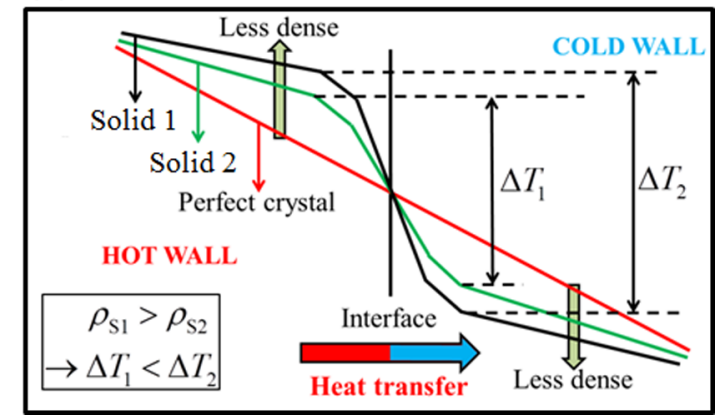

FIG. 1. Snapshot of the MD simulation domains with the domain size (a). Schematic of the differences of the temperature profiles between solid-liquid and solid-solid interfaces (b). Schematic of the effects of the crystal atomic density on the temperature jump on the GBs, in which $\rho_{\mathrm{S} 1}$ and $\rho_{\mathrm{S} 2}$ are the densities of solid 1 and solid 2, respectively, (c).

a nonlinear conjugate gradient algorithm was performed for the systems, whereby in-plane translations and atom deletion criteria were used to sample a large number of potential structures. ${ }^{41,42}$ The basic idea behind the energy minimization technique is to find the global minimum energy and thus, the most stable GB structures. Then, the atoms in the outermost layers were fixed to prevent atomic sublimation and to give the other atoms a bulk-like environment. The periodic conditions were only applied to the $\mathrm{X}$ and $\mathrm{Y}$ directions from this stage. Immediately adjacent to both sets of fixed atoms is a $1 \mathrm{~nm}$ thermal reservoir region, from which energy was added or removed to generate the thermal gradient and heat flux. The temperature of the left wall was kept higher than the right wall to generate thermal energy flux from the left to the right in the systems.

The simulation time step, $\tau$, was set at 1.0 fs. LAMMPS $^{43,44}$ was used for all MD simulations in the present study. All simulations were begun using the Maxwell-Boltzmann velocity distribution for all molecules at $300 \mathrm{~K}$. The NVT ensemble and Nose-Hoover thermostat were utilized to establish an equilibrium system at a temperature of $300 \mathrm{~K}$. Afterward, the Langevin thermostats were subjected to the hot side at $360 \mathrm{~K}$ and to the cold side at $280 \mathrm{~K}$ in order to induce heat flux through the models with the NVE ensemble. All simulations were performed for 12 ns: 2 ns to allow the system to reach an equilibrium state at room temperature, the next $4 \mathrm{~ns}$ ensured the system was steady in the presence of heat flux, and the last $6 \mathrm{~ns}$ for averaging the desired properties. Longer time averaging was also performed to confirm the convergence of the density and temperature profiles at steady state.

The Irving-Kirkwood (I-K) expression was utilized to calculate the heat flux vector for an $N$-particle system using the unity differential operator approximation as follows: ${ }^{45,46}$

$$
\begin{gathered}
J=\frac{1}{\operatorname{Vol}}\left\langle\sum_{i}^{N} V_{k}^{i}\left(E^{i}+P^{i}\right)+\sum_{i, j}^{N}\left(r_{k}^{j}-r_{k}^{i}\right) W^{i, j}\right\rangle, \\
E^{i}=\frac{1}{2} m^{i}\left(\left(V_{x}^{i}\right)^{2}+\left(V_{y}^{i}\right)^{2}+\left(V_{z}^{i}\right)^{2}\right), \\
W^{i, j}=\frac{1}{2}\left(V_{x}^{i} f_{x}^{i, j}+V_{y}^{i} f_{y}^{i, j}+V_{z}^{i} f_{z}^{i, j}\right),
\end{gathered}
$$

where the first term on the right-hand side of Eq. (2) represents the kinetic $\left(E^{i}\right)$ and potential energies $\left(P^{i}\right)$ carried by particle $i$, where the second term is the energy transferred to particle $i$ by force interactions $\left(W^{i, j}\right)$ with the surrounding particles. More specifically, $V_{k}^{i}$ is the velocity component of particle $i$ in the $k$-direction, where $k$ is the axes of the Cartesian coordinate system, $E^{i}$ is calculated by utilizing Eq. (3), $P^{i}$ is extracted from the EAM potential, and $\left(r_{k}^{j}-r_{k}^{i}\right)$ is the $k$ th component of the relative distance vector between particles $i$ and $j$. The $W^{i, j}$ term is given in Eq. (4), where $f_{k}^{i, j}$ is the intermolecular forces exerted on particle $i$ by particle $j$ in the Cartesian coordinate direction.

The GB energy per surface area, $\gamma_{\mathrm{GB}}$, can be obtained by using the following equation: 


$$
\gamma_{\mathrm{GB}}=\frac{\left\langle E_{\mathrm{GB}}\right\rangle-\left\langle E_{\mathrm{bulk}}\right\rangle}{A},
$$

where $\left\langle E_{\mathrm{GB}}\right\rangle$ and $\left\langle E_{\mathrm{bulk}}\right\rangle$ are the average potential energies (PEs) of atoms in the GB core and in the bulk, respectively, and $A$ is the cross section area on the $\mathrm{XY}$ plane in the supercell.

The heat transfer at an interface develops by the scattered and transmitted energy across the interfacial region. However, this interfacial region shows different characteristics at the solid-solid interface apart from the solid-liquid one. This is attributed to the different molecular interfacial structures developing difference in energy exchange. Therefore, it is important to clarify the differences in the thermal behaviors at solid-liquid and solid-solid interfaces, as described in Fig. 1(b). Typically, the molecules of a liquid, like those of a solid, are quite close together. However, solid molecules are held in fixed positions by intermolecular forces and exhibits long-range order. Hence, liquid molecules are freer to move. Under such conditions, the velocities of atoms at solid-liquid interfaces are exchanged much more easily to generate the temperature gradient. As a result, temperature drops abruptly at the solid-liquid interface and vice versa, gradual temperature jump develops in a finite region at the GBs. Fig. 1(c) shows a schematic representation of the variations of the temperature profile for the systems with different atomic densities. For a single crystal, the temperature is linearly distributed from the hot end to the cold end due to the continuity of the crystal structure. However, a temperature jump is observed with the presence of GB because the energy cannot be totally transmitted. Recently, Prasher modified the traditional welded AMM model by considering adhesion energy and molecular structure of interface. ${ }^{47} \mathrm{~A}$ more-closely packed atomic system can improve the interaction strength and energy transmission at interface, and thus, decrease the temperature jump and ITR.

\section{THE PROPER BIN SIZE FOR CORRECT MEASUREMENTS AT GRAIN BOUNDARIES}

To correctly calculate the ITR, we must first clarify the ambiguous definition of the bin size. This is because the divided bins are used not only to determine the temperature jump based on the temperature distribution along the channel length but also to analyze the molecular structures at the interface from density distributions. To the best of our knowledge, this is the first time that such an attempt has been made to modify the bin size. Typically, structural disorders have been observed only by atomic visualization. $13,14,39,48,49$ Therefore, using correct bins is necessary to provide insight into the interface characteristics.

The three types of crystal structures modeled in this study are shown in Fig. 2. The stacking sequence for the crystals in the $\mathrm{XY}$ plane is denoted on the top of Fig. 2 and the stacking sequence along the $\mathrm{Z}$ direction is presented at the bottom. The geometry of the crystals planes is characterized based on the lattice constant of a FCC copper crystal ( $a=3.61 \AA$ ). To determine the atomic density of a crystal, we utilized the definition of atomic surface density (ASD) as $\rho_{\mathrm{ASD}}=N / A$, where $N$ is the number of $\mathrm{Cu}$ atoms per surface area. Several articles suggested that the bin size should be comparable to the atomic diameter to be able to distinctly observe the characteristics of the amorphous medium. ${ }^{50,51}$ However, for a crystalline solid, planes are adjacently stacked with a similar spacing between layers corresponding to a particular crystal lattice. The ASD in each divided bin, which is perpendicular to the $\mathrm{Z}$ direction, is calculated resulting in the density distribution of the systems. Thus, an ASD of zero must be obtained in the gap between layers. The density profile thereby shows a periodic fluctuation corresponding to the crystal structure. To address this demand, the width of the divided bins should be equal to half of the distance between two layers. Under such conditions, one bin is located at the center of aligned solid atoms in a layer. The subsequent bin
(001)
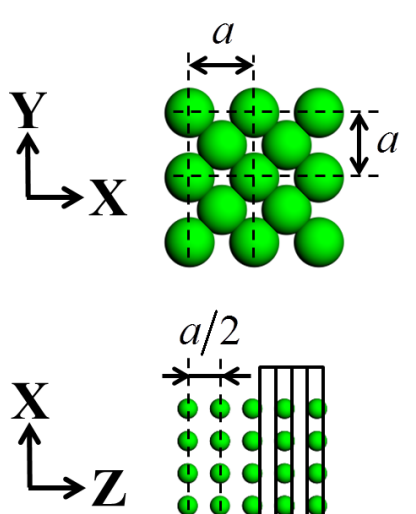
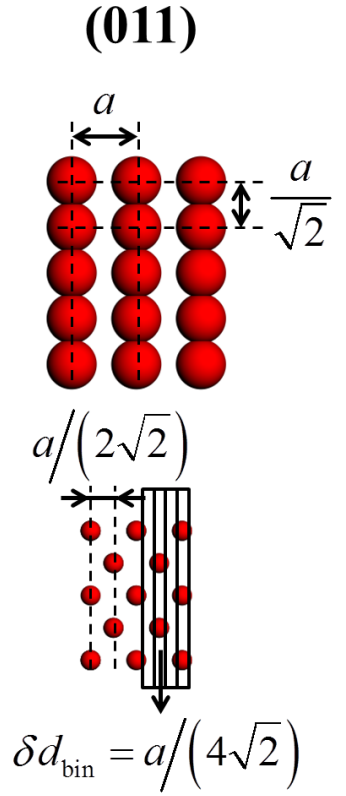

(111)

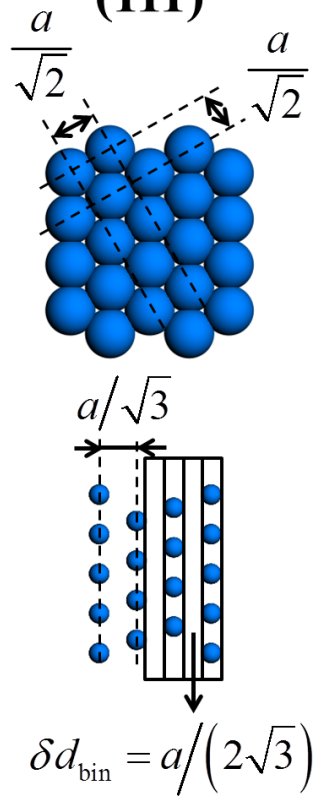

FIG. 2. Schematic of the stacking sequences for (001), (011), and (111) in the XY (top) and XZ planes (bottom). A proper bin size selection method is provided to accurately obtain the atomic density and temperature distributions for calculating the correct interfacial thermal resistance. 


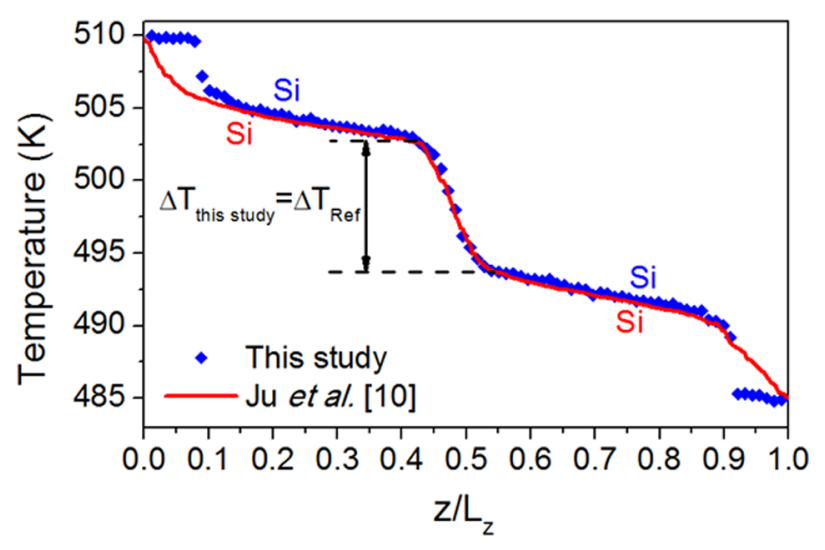

FIG. 3. Comparison of the obtained temperature profiles of twist boundary $\mathrm{Si}$ between using our approach of bin selection and from the recent study. ${ }^{10}$ Temperature and geometry configurations applied in our simulation are identical to that reference.

is positioned at the center of the gap between two solid layers leading to the zero ASD, as shown in the bottom of the figure. This definition was utilized throughout the entire analysis.

As a way to verify the accuracy of our approach for selecting the bin size, we performed simulation of heat transport across twist boundary of $\mathrm{Si}$ with identical temperature and geometry configurations to the recent study. ${ }^{10}$ The resulting temperature distributions showed good agreement not only in the bulk but also on the $\mathrm{Si}-\mathrm{Si}$ interface inducing a similar temperature jump (see Fig. 3). This agreement validated the proposed bin size selection. Furthermore, the gradual drop of temperature at GB, rather than a sharp drop, was confirmed. A similar trend has been reported for different solid-solid interfaces. ${ }^{11,12}$ Therefore, the heat transfer characteristics at such GBs need to be elucidated to provide better understanding and control of interfacial thermal transport.

\section{RESULTS AND DISCUSSION}

\section{A. Density and temperature profiles at grain boundaries}

The dynamics and structural properties of solid atoms are closely related to their concentration on the interface. Hence, the atomic density distribution of the systems studied here was evaluated to elucidate its impact on the thermal resistance at GBs by utilizing the developed bin size method and ASD definition. Figs. 4(a)-4(c) represent the plots of the density distribution along the $\mathrm{Z}$ direction for the cases studied. The average ASD for a system can be expressed as $\left\langle\rho_{\mathrm{ASD}}\right\rangle=\left(\rho_{\mathrm{ASD}}^{\mathrm{G} 1}+\rho_{\mathrm{ASD}}^{\mathrm{G} 2}\right) / 2$, where $\rho_{\mathrm{ASD}}^{\mathrm{G} 1}$ and $\rho_{\mathrm{ASD}}^{\mathrm{G} 2}$ are the ASDs in grain 1 and grain 2 , respectively, as shown in Table I. It is noted that the ASD in each grain is calculated by considering the slab bin containing the atomic layer in the bulk. In addition, $\left\langle\rho_{\mathrm{ASD}}\right\rangle$ is used as a factor in investigating the effects of the system atomic density on the thermal transport. A similar assumption of the system atomic density was developed in the report of Prasher. ${ }^{47}$ As expected, the density profiles showed periodic fluctuations in the bulk region due to the repetitive ordering of solid molecules. Interestingly, the structure at the boundary composed of the (001) and (111) crystal surfaces exhibited no disorder, as seen in Fig. 4(c). However, a disturbance was significantly found in the other cases which contained the (011) surface model. This can be interpreted that the (011) surface is less close-packed in the FCC crystal leading to the less strong attraction force between atoms, and thus atoms in this plane move easily, which would cause the structural disorders at GB. (a)

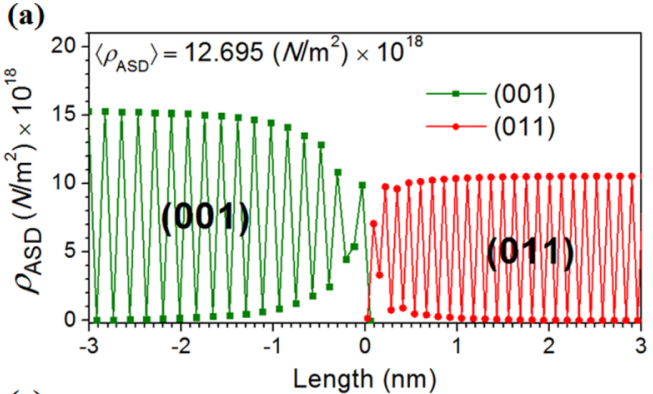

(c)

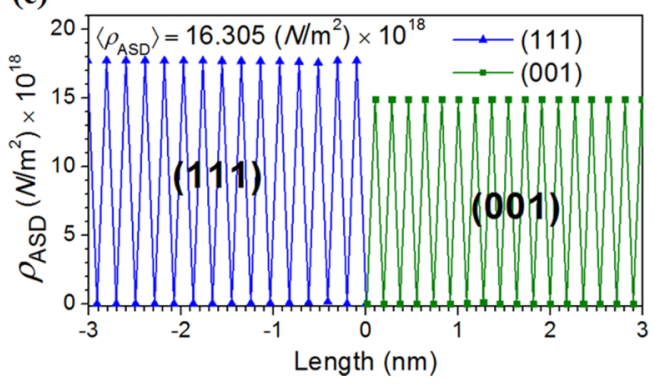

(b)

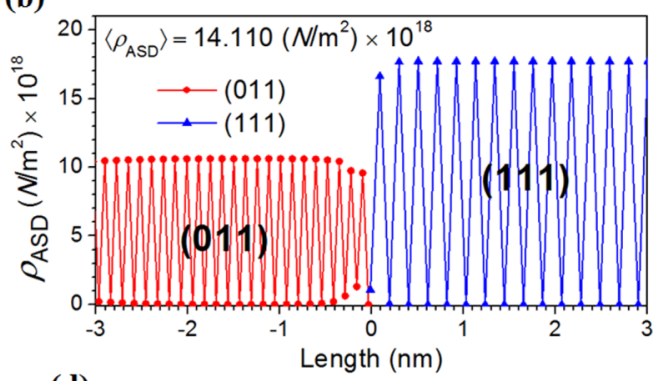

(d)

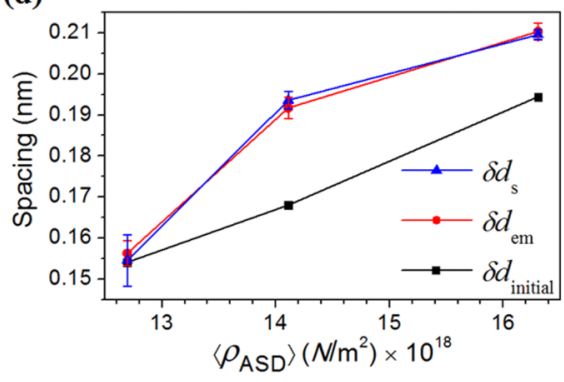

FIG. 4. Atomic density distributions along the $\mathrm{Z}$ direction for several cases of crystal atomic density ((a)-(c)). Spacing between two grains calculated from the arithmetic mean, after energy minimization, and after the systems reaches steady state (d). The average interfacial spacing obtained from three dependent simulations for each case is plotted with error bars showing the standard deviation in (c). 
TABLE I. Atomic surface density corresponding to each crystal, $\rho_{\mathrm{ASD}}$, and the system's average ASD, $\left\langle\rho_{\mathrm{ASD}}\right\rangle$, for the cases studied in this work.

\begin{tabular}{lcc}
\hline \hline Case & $\rho_{\text {ASD }}\left(N / \mathrm{m}^{2}\right)$ & $\left\langle\rho_{\text {ASD }}\right\rangle\left(N / \mathrm{m}^{2}\right)$ \\
\hline$(011)$ & $10.609 \times 10^{18}$ & N/A \\
$(001)$ & $14.890 \times 10^{18}$ & N/A \\
$(111)$ & $17.723 \times 10^{18}$ & N/A \\
$(001) /(011)$ & $\mathrm{N} / \mathrm{A}$ & $12.695 \times 10^{18}$ \\
$(011) /(111)$ & $\mathrm{N} / \mathrm{A}$ & $14.110 \times 10^{18}$ \\
$(111) /(001)$ & $\mathrm{N} / \mathrm{A}$ & $16.305 \times 10^{18}$ \\
\hline \hline
\end{tabular}

On the other hand, the interfacial spacing between two grains after the energy minimization, $\delta d_{\mathrm{em}}$, and after the equilibrium state, $\delta d_{\mathrm{s}}$, were calculated and compared to $\delta d_{\text {initial}}$, as plotted in Fig. 4(d). A deviation between $\delta d_{\text {initial }}$ and the other values was observed. Meanwhile, the differences between the $\delta d_{\mathrm{em}}$ and $\delta d_{\mathrm{s}}$ values were negligible. This implies that there was no mechanical stress applied to fixing both ends after the energy minimization. In other words, the bulk-like environment was maintained.

The temperature distributions are presented in Fig. 5(a). We carefully studied the probable effects from the selected bin size on the measured quantities, specifically on the measured local temperatures. For such a case, we properly tested the existence of the local thermal equilibrium in each of the statistical averaging bin in order to validate that the local temperature can be defined for the case of the bin size which is on the order of the crystal layer spacing. The results showed that the number of molecules is sufficient and the molecular velocities develop the wellknown Maxwell-Boltzmann distribution at corresponding temperatures. Most importantly, we further compared the results of small size bins with the results obtained using larger size bins. Comparison showed agreement, which ultimately validates that the presented results are free from any bin size related effects. It is noted that the temperatures' averages in the bins located at the gaps were removed due to the limited number of solid particles for averaging. An artificial thermal resistance was observed between the thermostat applied region and the free thermostat applied region. ${ }^{26}$ This can be neglected when computationally studying nanoscale thermal transport. Meanwhile, a significant temperature jump was found at the interface due to the dissimilarity in the crystal structures. In addition, the temperature decreased gradually at the GB inducing the thermal slopes that were verified previously. Figure 5(b) shows a close-up of the temperature profile at the boundary for all cases studied. To obtain $\Delta T$, both the temperature distributions in the hot and cold domain were characterized by least-squares fits. In this way, $\Delta T$ was achieved from the difference between these two fits at the interface, as denoted by the arrows. The dissimilarity $\left\langle\rho_{\mathrm{ASD}}\right\rangle$ between the systems is the cause of the different $\Delta T$ values obtained. Specifically, the more substantial the atomic density

(a)

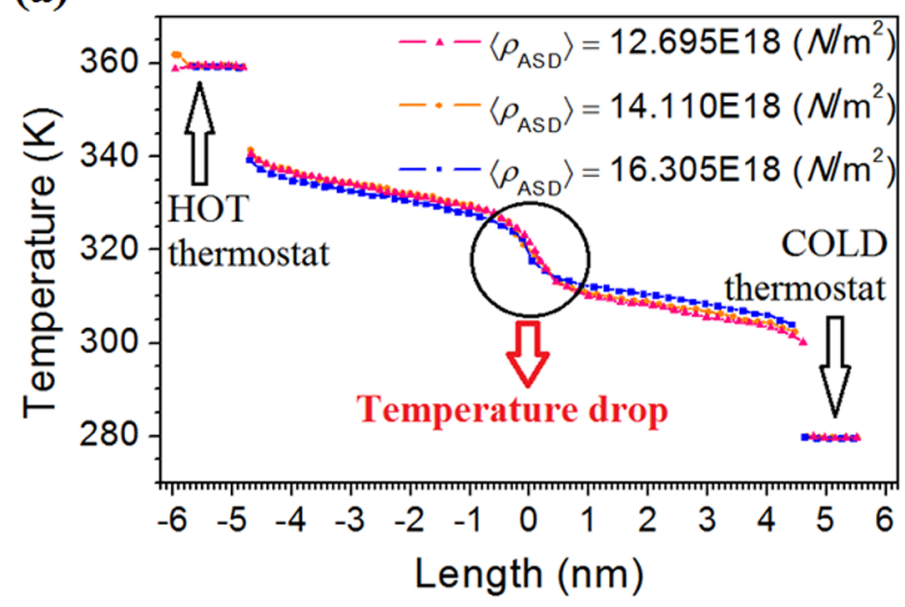

(b)

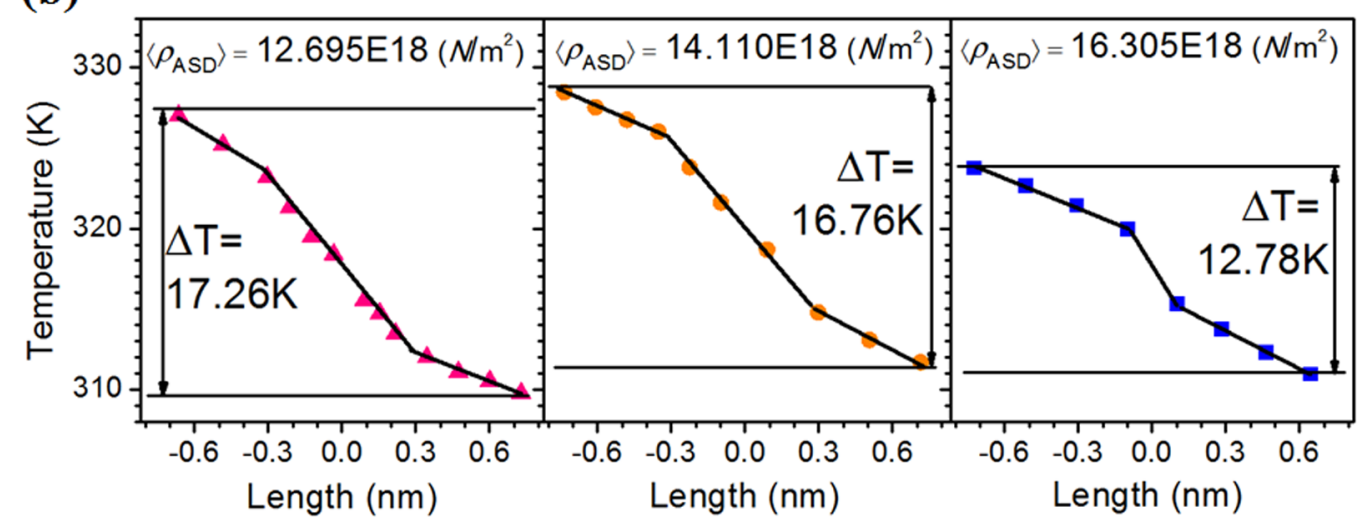

FIG. 5. Temperature profiles along the heat transfer direction for the cases investigated in this study (a). Close-up of the temperature profiles showing the presence of temperature slopes at GBs (b). 
(a)

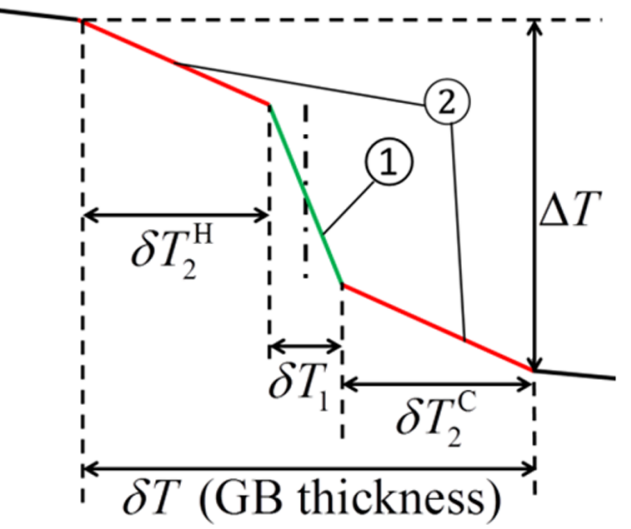

(b)

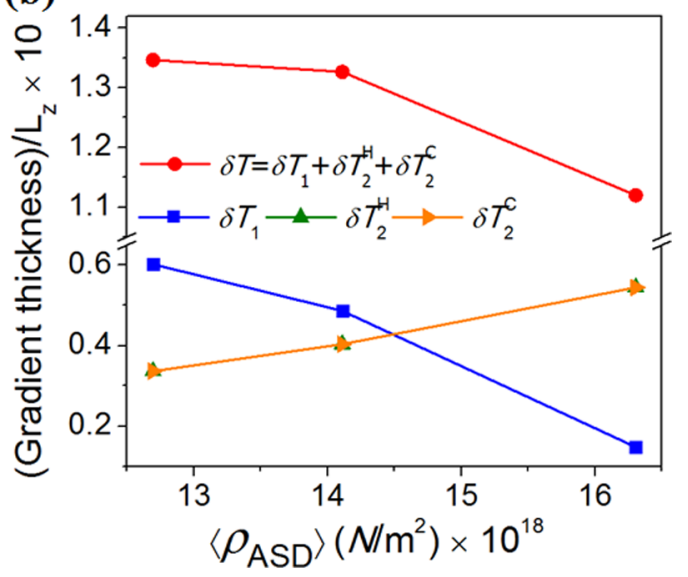

FIG. 6. A schematic definition of the GB thickness (a). Effects of the atomic density on the thickness of temperature gradients within the interface region (b)

of the system, the less the temperature jump at the GB and vice versa. The origin of this phenomenon is the change of the probability of intermolecular interactions between $\mathrm{Cu}$ atoms, which is strongly affected by the atomic concentration at the boundary. It is clear that an increase of the concentration of atoms enhances the momentum and heat transfer. In other words, by utilizing the system in which the planes are highly close-packed, the chance for phonon transmission can be improved. This results in a reduction of the ITR. Interestingly, insight into the temperature jump region significantly showed not only one, but three distinct temperature gradients. This must be actively investigated to obtain correct interfacial characteristics.

To thoroughly understand the thermal behaviors at the $\mathrm{GB}$, a schematic representation is shown in Fig. 6(a). It was assumed that the temperature slope crosses over the interface $(z=0)$, namely, region 1 , having a thickness of $\delta T_{1}$. Region 1 is additionally confined by region 2 , which is the other two temperature slopes exhibiting the thicknesses of $\delta T_{2}^{\mathrm{H}}$ and $\delta T_{2}^{\mathrm{C}}$ in the hot wall and in the cold wall, respectively. As a result, the width of region 2 is estimated as $\delta T_{2}=\delta T_{2}^{\mathrm{H}}+\delta T_{2}^{\mathrm{C}}$. Therefore, the GB thickness, $\delta T$, can be estimated as the sum of the thicknesses of regions 1 and 2. The thicknesses of the temperature gradients at the interface were computed and are plotted in Fig. 6(b). For a specific system, $\delta T_{2}^{\mathrm{H}}$ and $\delta T_{2}^{\mathrm{C}}$ were found to be similar. However, MD simulation results revealed that $\delta T_{1}$ showed an inverse tendency with $\delta T_{2}$ when increasing $\left\langle\rho_{\mathrm{ASD}}\right\rangle$. Interestingly, $\delta T$ was not constant as it decreased monotonically with the enhanced $\left\langle\rho_{\mathrm{ASD}}\right\rangle$. Not only the gradient thickness but also the temperature profile shown in Fig. 5(b) scrutinizes the discrepancies between these regions, in which the temperature gradient in region 1 is greater than in region 2. Thus, it can be concluded that the dynamics of the atoms belonging to the different regions are different.

From the observed results, we can infer the reasons of the three temperature gradients at the interface: the different properties of heat carriers on the two sides of the interface inducing the phonon mismatch and phonon backscattering, ${ }^{7}$ and atomic forces between dissimilar structures. Thus, it is able to explain the GB thickness variation with the system atomic density. A high-coherent interface increases the energy transmission and decreases the phonon mismatch, leading to the reduced the backscattering of phonons. This is the source of the decreased GB thickness when using the system having closely packed planes. In contrast, the rough interface caused by random atomic roughness promotes the incoherent phonon scattering and decrease the phonon transmission, causing the enhanced GB thickness. It should be noted that the energy
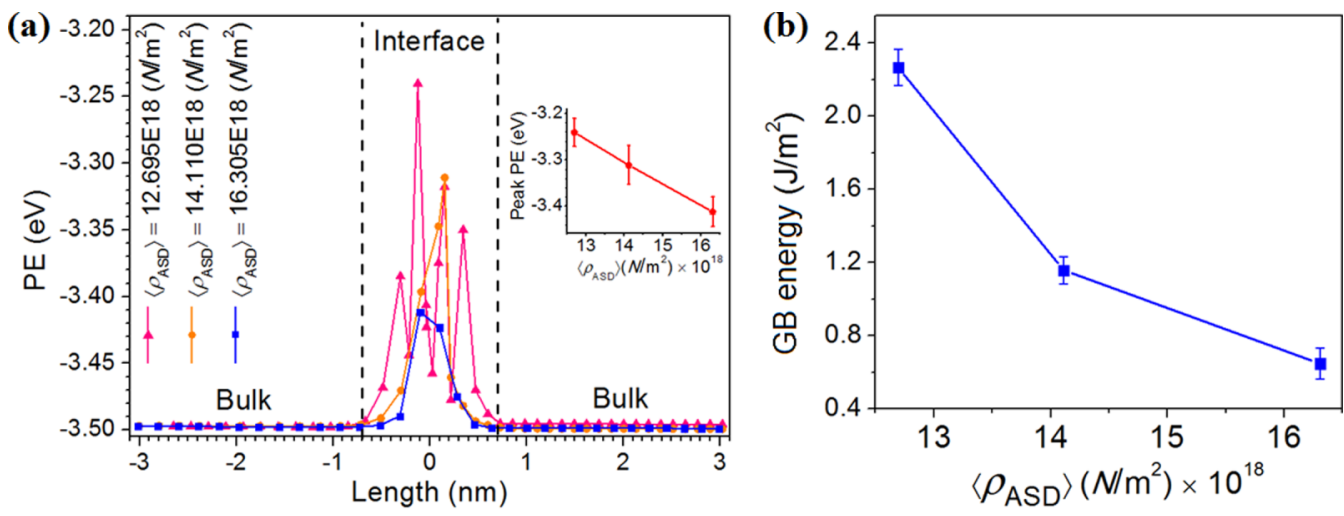

FIG. 7. Potential energy distribution along the heat transfer direction where the inset shows the system atomic density dependence on the potential energy peak (a). GB energy as a function of atomic density (b). The average GB energy obtained from three dependent simulations for each case is plotted with error bars showing the standard deviation in (b). 
TABLE II. Grain boundary thickness, $\delta T$, obtained from the temperature distribution, potential energy distribution, and Brutzel's definition. ${ }^{53}$

\begin{tabular}{|c|c|c|c|c|c|}
\hline \multirow[b]{2}{*}{$\left\langle\rho_{\mathrm{ASD}}\right\rangle\left(N / \mathrm{m}^{2}\right)$} & \multirow{2}{*}{$\begin{array}{c}\delta T_{2}^{\mathrm{H}}\left(=\delta T_{2}^{\mathrm{C}}\right) \\
(\mathrm{nm})\end{array}$} & \multicolumn{3}{|c|}{$\delta T(\mathrm{~nm})$ obtained from } & \multirow{2}{*}{$\begin{array}{l}{[(\mathrm{a})-(\mathrm{b})] / 2} \\
\quad(\mathrm{~nm})\end{array}$} \\
\hline & & distribution & PE distribution (a) & Brutzel's definition $^{53}$ (b) & \\
\hline $12.695 \times 10^{18}$ & 0.34 & 1.35 & 1.37 & 0.77 & 0.30 \\
\hline $14.110 \times 10^{18}$ & 0.42 & 1.33 & 1.33 & 0.42 & 0.46 \\
\hline $16.305 \times 10^{18}$ & 0.49 & 1.12 & 1.08 & 0.22 & 0.43 \\
\hline
\end{tabular}

transmission might be improved if regular-shape patterned (i.e., periodic-rough) interfaces are generated. ${ }^{52}$

\section{B. Effects of density on thermal transport across the grain boundary}

Intermolecular interaction is an important property affecting the solid-solid interface. In this study, the probability of interfacial interactions is influenced by changing the crystal atomic densities. In such cases, investigating the effects of interfacial geometry, especially the concentration of atoms at the interface on the ITR is necessary. The temperature and density profiles across the GB were corrected and examined using our proposed method. Therefore, we believe that the ITR values obtained from our MD simulations are accurate.

First, we made an effort to understand the relationship between the interfacial geometry and GB energy. The GB energy can be calculated by using Equation (5). PE distributions along the heat transfer direction for the corresponding systems are plotted in Fig. 7(a). The results showed significant differences at the interface from the bulk. Specifically, the PE within the bulk domain was steady and converged on the bulk value. Meanwhile, the PE close to the boundary became dominant and showed fluctuations due to the different coordination numbers from the bulk atoms. These findings newly reinforce the importance of structural disturbances, in which atoms demonstrated dissimilar dynamics properties compared to the bulk. In addition, the inset in Fig. 7 (a) describes the $\left\langle\rho_{\text {ASD }}\right\rangle$ dependence on the PE peak. A high-mismatch interface induced low attractive forces between atoms composing at the GB. It may be useful to remind the reader that the bin size utilized to obtain the PE distribution was identical to that applied to achieve the density and temperature distributions.

In Sec. IV A, the GB thickness was considered as a sum of the thickness of temperature slopes on the interface. Furthermore, the PE profiles revealed that the dynamic behaviors of interface atoms are different from the bulk atoms inducing different potential energies. Thus, the GB thickness is additionally defined as the width between two points where the PE differs from the average value in the bulk. As shown in Table II, the GB thickness obtained from PE profiles exhibited excellent agreement with that obtained from the temperature profiles. This agreement furthermore validates the proper binning. On the other hand, Brutzel and Vincent-Aublant provided criteria to predict the interface thickness by using the PE distribution, where the boundary atom's PE differs from the bulk average by a certain value, i.e., $0.05 \mathrm{eV}^{53} \mathrm{~A}$ comparison of the obtained $\delta T$ between the Brutzel's assumption and our assumption is presented in Table II showing notable differences. Interestingly, this deviation was found to be the thickness of region 2 for every case studied. Hence, it is evident that region 2 was omitted in Ref. 53 due to the inaccurate bin size selected. Moreover, Fig. 7(b) shows a plot of $\gamma_{\mathrm{GB}}$ with the variations of $\left\langle\rho_{\mathrm{ASD}}\right\rangle$. It was found that an increase of $\left\langle\rho_{\mathrm{ASD}}\right\rangle$ results in a reduced $\gamma_{\mathrm{GB}}$. This is because the geometries strongly affect the coherence at the GBs. An interface reaching a lower GB energy suggests a less mismatch.

The temperature profile across the GB was corrected previously. This provides an accurate calculation for the
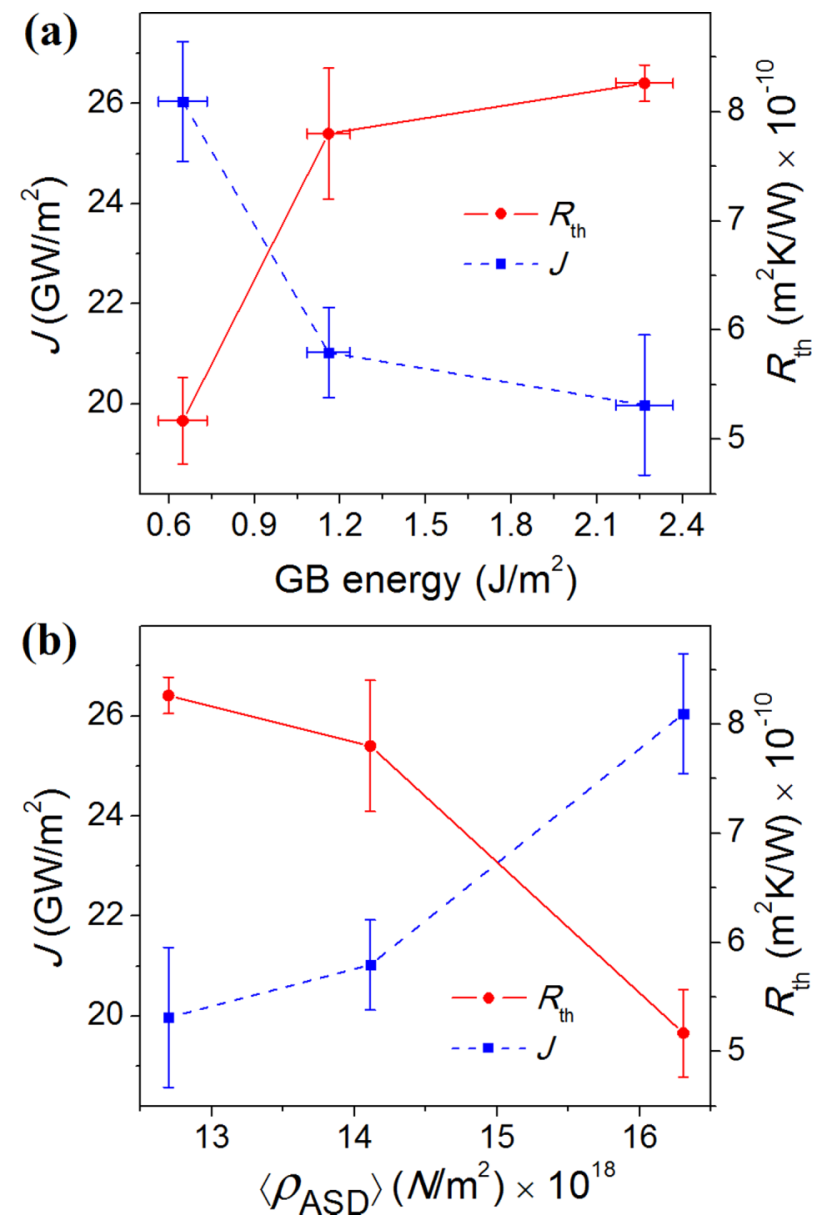

FIG. 8. Heat flux and ITR as functions of the (a) GB energy and the (b) system atomic density. The average heat flux and ITR obtained from three dependent simulations for each case is plotted with error bars showing the standard deviation in (a) and (b). 
temperature jump and ITR based on Equation (1). Meanwhile, the heat flux through the system can be obtained by utilizing Equation (2). The complexity in the case of solid-solid interfaces results from the wide variety of defects that can form when the interface is crystalline on both sides. The composition or degree of order may be dramatically different across the interface. Hence, we considered the effects of $\gamma_{\mathrm{GB}}$ and $\left\langle\rho_{\mathrm{ASD}}\right\rangle$ on the ITR and heat flux, as shown in Fig. 8. It is well-known that a reduction of the resulting ITR in the system improves the thermal transport efficiency. This implies that the heat flux increases with decreasing ITR. We observed results similar to recent studies ${ }^{12,53}$ as a system resulting in a high $\gamma_{\mathrm{GB}}$ indicates high mismatch at the interface, leading to the elevated ITR and reduced heat flux. In other words, by utilizing system with a high $\left\langle\rho_{\mathrm{ASD}}\right\rangle$, the probability for the interactions at the interface can be enhanced, leading to improved momentum transfer and heat transport. This reduces the ITR and increases the heat flux through the system.

To better understand the mechanism of crystal atomic density dependence on ITR, we calculated the vibrational density of states (VDOS), i.e., number of vibrational modes per unit volume and frequency, by performing a Fourier transform of velocity autocorrelation function (VACF). The VACF was obtained from equilibrium MD simulation. We calculated the VDOS of $\mathrm{Cu}$ atoms of the two different grains of the interfaces. The resulting VDOS are shown in Fig. 9. We observed spectrum change at the interfaces of different crystal structures. It also supports the conclusion that the atomic density strongly affects the interfacial thermal

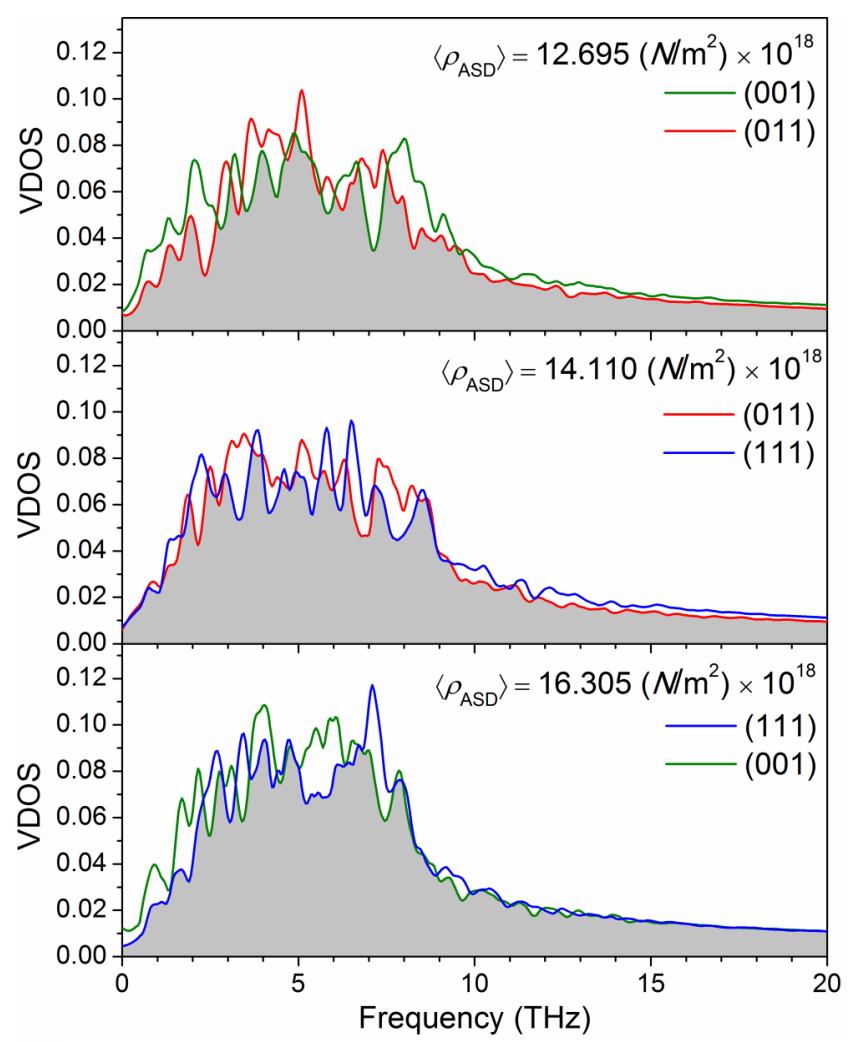

FIG. 9. VDOS of $\mathrm{Cu}$ atoms in the two grains forming at interface for all cases studied in this work. The gray area illustrates the overlap between VDOS. transport. It is well-known that a good overlap between the spectrums implies a strong vibrational coupling and, consequently, low ITR. Our results revealed that the overlap decreased with decreasing crystal atomic density. In other words, the presence of structural disorders at solid-solid interface reduced the overlap between VDOS. As a result, the interfacial conductance decreased.

\section{CONCLUSIONS}

By using MD simulations, we corrected the temperature profiles across the GBs to accurately calculate the ITR. When examining nanoscale heat transfer, the bin size used to average the desired data must be defined carefully. Otherwise, ambiguities of thermal behaviors at the interface may result. To address this, we introduced a proper bin size that is on the order of the crystal layer spacing to achieve correct temperature and atomic density distributions. The solid-solid interfaces of $\mathrm{Cu}$ having (001), (011), and (111) orientations were investigated in the present study. The following results were obtained.

1. The temperature decreases gradually at the solid-solid interface in place of the well-known sudden drop observed at the solid-liquid interface. This is due to the amorphous structure of the liquid state that allows liquid molecules to move freely, leading to the momentum of atoms being much easier to be transferred from the interface to the bulk. Consequently, three distinct temperature gradients develop at the GB showing different behaviors than the one obtained in the bulk solid. The observed temperature discontinuities at the boundary of grains were developed as a combined effect of the phonon mismatch, phonon backscattering, and atomic forces between dissimilar structures at the GB.

2. The GB thickness, which was calculated by utilizing either the temperature profiles or the potential energy distributions, is highly dependent on the system atomic density.

3. The atomic packing of a system was found to be the key factor affecting the atomic structures and thermal properties at the interface. By using a system in which the planes are highly close-packed, the probability for interactions between solid particles at the GBs was improved. Under such conditions, the overlap between VDOS of atoms forming at interfaces increased. As a result, the temperature jump, the GB energy, and the ITR were reduced, whereas the heat flux was enhanced.

In conclusion, the crystal atomic density is the primary parameter for structural disorders and heat flow properties at the GBs. A comprehensive understanding of temperature characteristics and thermal transport at solid-solid interfaces was formulated by utilizing proper binning. This study provides useful information for the design of nanostructured devices and nano-engineered materials aimed at optimizing operational efficiency. 


\section{ACKNOWLEDGMENTS}

This research was supported by the Basic Science Research Program through the National Research Foundation of Korea (NRF) funded by the Ministry of Education (Grant No. NRF-2014R1A1A2057147).

${ }^{1}$ P. E. Hopkins, ISRN Mech. Eng. 2013, 1.

${ }^{2}$ D. G. Cahill, P. V. Braun, G. Chen, D. R. Clarke, S. Fan, K. E. Goodson, P. Keblinski, W. P. King, G. D. Mahan, and A. Majumdar, Appl. Phys. Rev. 1, 011305 (2014).

${ }^{3}$ J. M. Howe, Interfaces in Materials: Atomic Structure, Thermodynamics and Kinetics of Solid-Vapor, Solid-Liquid and Solid-Solid Interfaces (WileyInterscience, 1997)

${ }^{4}$ G. L. Pollack, Rev. Mod. Phys. 41, 48 (1969).

${ }^{5}$ W. Little, Can. J. Phys. 37, 334 (1959).

${ }^{6}$ E. T. Swartz and R. O. Pohl, Rev. Mod. Phys. 61, 605 (1989).

${ }^{7}$ P. Schelling, S. Phillpot, and P. Keblinski, J. Appl. Phys. 95, 6082 (2004).

${ }^{8}$ K. Ikeda, K. Yamada, N. Takata, F. Yoshida, H. Nakashima, and N. Tsuji, Mater. Trans. 49, 24 (2008).

${ }^{9}$ D. Wolf and S. Phillpot, Mater. Sci. Eng.: A 107, 3 (1989).

${ }^{10}$ S. Ju and X. Liang, J. Appl. Phys. 113, 053513 (2013).

${ }^{11}$ J. Crocombette and L. Gelebart, J. Appl. Phys. 106, 083520 (2009).

${ }^{12}$ T. Chen, D. Chen, B. H. Sencer, and L. Shao, J. Nucl. Mater. 452, 364 (2014).

${ }^{13}$ T. Watanabe, B. Ni, S. R. Phillpot, P. K. Schelling, and P. Keblinski, J. Appl. Phys. 102, 063503 (2007).

${ }^{14}$ R. J. Stevens, L. V. Zhigilei, and P. M. Norris, Int. J. Heat Mass Transfer 50, 3977 (2007).

${ }^{15}$ S. Merabia and K. Termentzidis, Phys. Rev. B 86, 094303 (2012).

${ }^{16}$ P. Schelling, S. Phillpot, and P. Keblinski, Appl. Phys. Lett. 80, 2484 (2002).

${ }^{17}$ C. Kimmer, S. Aubry, A. Skye, and P. K. Schelling, Phys. Rev. B 75, 144105 (2007).

${ }^{18}$ E. E. Helgee and A. Isacsson, Phys. Rev. B 90, 045416 (2014).

${ }^{19}$ Z. Tian, B. White, Jr., and Y. Sun, Appl. Phys. Lett. 96, 263113 (2010).

${ }^{20}$ Z. Zheng, X. Chen, B. Deng, A. Chernatynskiy, S. Yang, L. Xiong, and Y. Chen, J. Appl. Phys. 116, 073706 (2014).

${ }^{21}$ P. Schelling and S. Phillpot, J. Appl. Phys. 93, 5377 (2003).

${ }^{22}$ N. Stojanovic, D. Maithripala, J. Berg, and M. Holtz, Phys. Rev. B 82, 075418 (2010).
${ }^{23}$ J. C. Duda, C. J. Kimmer, W. A. Soffa, X. W. Zhou, R. E. Jones, and P. E. Hopkins, J. Appl. Phys. 112, 093515 (2012).

${ }^{24}$ Z. Liang, W. J. Evans, and P. Keblinski, J. Chem. Phys. 141, 014706 (2014).

${ }^{25}$ G. Balasubramanian and I. K. Puri, Appl. Phys. Lett. 99, 013116 (2011).

${ }^{26}$ M. Barisik and A. Beskok, J. Comput. Phys. 231, 7881 (2012).

${ }^{27}$ T. Q. Vo and B. Kim, Int. J. Precis. Eng. Manuf. 16, 1341 (2015).

${ }^{28}$ B. Kim, Chem. Phys. Lett. 554, 77-81 (2012).

${ }^{29}$ B. H. Kim, A. Beskok, and T. Cagin, J. Chem. Phys. 129, 174701 (2008)

${ }^{30}$ M. Barisik and A. Beskok, Int. J. Therm. Sci. 77, 47 (2014).

${ }^{31}$ A. Pham, M. Barisik, and B. Kim, J. Chem. Phys. 139, 244702 (2013).

${ }^{32}$ T. Zushi, K. Ohmori, K. Yamada, and T. Watanabe, Phys. Rev. B 91, 115308 (2015).

${ }^{33}$ S. Aubry, C. J. Kimmer, A. Skye, and P. K. Schelling, Phys. Rev. B 78, 064112 (2008).

${ }^{34}$ B. C. Daly, H. J. Maris, K. Imamura, and S. Tamura, Phys. Rev. B 66, 024301 (2002).

${ }^{35}$ A. Bodapati, P. K. Schelling, S. R. Phillpot, and P. Keblinski, Phys. Rev. B 74, 245207 (2006).

${ }^{36}$ P. K. Schelling, S. R. Phillpot, and P. Keblinski, Phys. Rev. B 65, 144306 (2002).

${ }^{37}$ Y. Hua and B. Cao, Int. J. Heat Mass Transfer 78, 755 (2014).

${ }^{38}$ D. Kraemer, B. Poudel, H. Feng, J. C. Caylor, B. Yu, X. Yan, Y. Ma, X. Wang, D. Wang, and A. Muto, Nat. Mater. 10, 532 (2011).

${ }^{39}$ H. Van Swygenhoven, D. Farkas, and A. Caro, Phys. Rev. B 62, 831 (2000).

${ }^{40}$ J. Mei, J. Davenport, and G. Fernando, Phys. Rev. B 43, 4653 (1991)

${ }^{41}$ M. Tschopp and D. McDowell, Philos. Mag. 87, 3871 (2007).

${ }^{42}$ M. Tschopp and D. McDowell, Philos. Mag. 87, 3147 (2007)

${ }^{43}$ S. Plimpton, J. Comput. Phys. 117, 1 (1995).

${ }^{44}$ See http://lammps.sandia.gov/ for LAMMPS.

${ }^{45}$ J. Irving and J. G. Kirkwood, J. Chem. Phys. 18, 817 (1950).

${ }^{46}$ B. Todd, P. J. Daivis, and D. J. Evans, Phys. Rev. E 51, 4362 (1995).

${ }^{47}$ R. Prasher, Appl. Phys. Lett. 94, 041905 (2009).

${ }^{48}$ A. France-Lanord, S. Merabia, T. Albaret, D. Lacroix, and K. Termentzidis, J. Phys.: Condens. Matter 26, 355801 (2014).

${ }^{49}$ H. Somekawa and T. Mukai, Mater. Lett. 76, 32 (2012).

${ }^{50}$ B. H. Kim, A. Beskok, and T. Cagin, Microfluid. Nanofluid. 9, 31 (2010).

${ }^{51}$ T. Vo, B. Park, C. Park, and B. Kim, J. Mech. Sci. Technol. 29, 1681 (2015).

${ }^{52}$ S. Merabia, Phys. Rev. B 89, 054309 (2014).

${ }^{53}$ L. V. Brutzel and E. Vincent-Aublant, J. Nucl. Mater. 377, 522 (2008). 